Research Article

\title{
Oxidized DJ-1 Levels in Urine Samples as a Putative Biomarker for Parkinson's Disease
}

\author{
Jihoon Jang, ${ }^{1}$ Soyeon Jeong, ${ }^{1,2}$ Sung Ik Lee, ${ }^{3}$ Wongi Seol $\mathbb{D}^{2},{ }^{2}$ Hyemyung Seo $\mathbb{D},{ }^{1}$ \\ Ilhong Son $\left(\mathbb{1},,^{2,3}\right.$ and Dong Hwan Ho ${ }^{2}{ }^{2}$ \\ ${ }^{1}$ Department of Molecular and Life Sciences, Hanyang University, Ansan-si, Gyeonggido, Republic of Korea \\ ${ }^{2}$ InAm Neuroscience Research Center, Sanbon Medical Center, College of Medicine, Wonkwang University, \\ Sanbon-ro, Gunpo-si, Gyeonggido, Republic of Korea \\ ${ }^{3}$ Department of Neurology, Sanbon Medical Center, College of Medicine, Wonkwang University, Sanbon-ro, \\ Gunpo-si, Gyeonggido, Republic of Korea
}

Correspondence should be addressed to Ilhong Son; sonih@wku.ac.kr and Dong Hwan Ho; ethan2887@gmail.com

Received 27 November 2017; Accepted 25 March 2018; Published 14 May 2018

Academic Editor: Kumar Vaibhav

Copyright (c) 2018 Jihoon Jang et al. This is an open access article distributed under the Creative Commons Attribution License, which permits unrestricted use, distribution, and reproduction in any medium, provided the original work is properly cited.

\begin{abstract}
Parkinson's disease (PD) is the second most common neurodegenerative disease. Oxidative stress is the most critical risk factor for neurodegenerative diseases, including Alzheimer's disease (AD) and Huntington's disease (HD). Numerous reports have demonstrated that oxidative stress aggravates cytotoxicity in dopaminergic neurons and accelerates the formation of protein inclusions. In addition, oxidative stress, such as 4-hydroxynonenal (HNE), oxidized protein, and dopamine quinone, are related to PD progression. DJ-1 is a PD-causative gene, and it plays a pivotal role as a sensor and eliminator of oxidative stress. Several studies have shown that oxidized DJ-1 (OxiDJ-1) formation is induced by oxidative stress. Hence, previous studies suggest that oxidized DJ-1 could be a biomarker for PD. We previously reported higher DJ-1 levels in Korean male PD patient urine exosomes than male non-PD controls. We speculate that OxiDJ-1 levels in PD patient urine might be higher than that in non-PD controls. In this study, we established an ELISA for OxiDJ-1 using recombinant DJ-1 treated with $\mathrm{H}_{2} \mathrm{O}_{2}$. Using Western blot assay and ELISA, we confirmed an increase of OxiDJ-1 from HEK293T cells treated with $\mathrm{H}_{2} \mathrm{O}_{2}$. Using our ELISA, we observed significantly higher, 2-fold, OxiDJ-1 levels in the urine of Korean PD patients than in non-PD controls.
\end{abstract}

\section{Introduction}

Parkinson's disease is the second most common neurodegenerative disease, and its primary symptoms include tremor, bradykinesia, and rigidity. The progression of $\mathrm{PD}$ is associated with an increase in reactive oxygen species (ROS), including superoxide anion, hydrogen peroxide, hydroxyl radicals, singlet oxygen, and lipid peroxyl radicals [1]. Previous studies have revealed that 4-hydroxynonenal (HNE), a by-product of lipid peroxidation, is one of the most bioactive aldehydes, and oxidative stress initiates its production [2]. It has been implicated that oxidative modification of proteins is accelerated by increase of ROS $[3,4]$. The synthesis of dopamine quinone (DAQ) is also mediated by the oxidation of dopamine, thereby initiating mitochondrial damage and generation of
DAQ-conjugated cytosolic adducts $[5,6]$. These features are closely related to $\mathrm{PD}$ pathology.

$D J-1$ is known as a PD-causative gene, and its function is associated with oxidative stress in PD [7]. A number of reports have demonstrated that DJ-1 senses ROS in the cytosol and conjugates ROS oxygen groups on its cysteine-106 residue $[8,9]$. Loss of ROS scavenger function in DJ-1 showed enhanced cytotoxicity and increase of $\alpha$-synuclein ( $\alpha$-syn) aggregates, which are known as a culprit of PD-pathogenesis [10-12]. Interestingly, a previous report proposed that oxidized DJ-1 (OxiDJ-1) would be an efficient biomarker for PD diagnosis [13]. Levels of DJ-1 in biofluids, such as cerebrospinal fluid (CSF), whole blood, plasma, serum, saliva, and urine, were investigated as a biomarker for PD (Table 1). There were higher levels of OxiDJ-1 in the erythrocytes of PD patients than 
TABLe 1: Summary of clinical studied examining DJ-1 and OxiDJ-1 as possible biomarkers.

\begin{tabular}{|c|c|c|c|}
\hline Material & Method & Outcome feature & Reference \\
\hline CSF & $\mathrm{WB}^{\mathrm{a}}$ & DJ-1 levels were significantly higher in PD. & {$[15]$} \\
\hline Plasma & WB & DJ-1 levels in PD were higher than those in controls. & {$[16]$} \\
\hline Serum & ELISA $^{\mathrm{b}}$ & DJ-1 shows similar levels in PD and in controls. & {$[17]$} \\
\hline $\mathrm{RBC}$ & ELISA & $\begin{array}{l}\text { OxiDJ-1 levels in unmedicated PD were higher than } \\
\text { medicated PD or healthy control. }\end{array}$ & [13] \\
\hline CSF & Luminex assay & DJ-1 levels were higher in PD than in control or AD. & {$[18]$} \\
\hline Plasma & Luminex assay & DJ-1 was not a suitable biomarker of PD. & {$[19]$} \\
\hline Saliva & Luminex assay & DJ-1 concentration was higher in PD. & {$[20]$} \\
\hline CSF & Luminex assay & DJ-1 levels in PD were lower than in controls. & [20] \\
\hline Whole blood & 2D-PAGE, WB & DJ-1 levels were changed in the late stage of PD. & [21] \\
\hline CSF & ELISA & $\begin{array}{c}\text { DJ-1 levels did not change among Parkinsonian } \\
\text { syndromes. }\end{array}$ & {$[22]$} \\
\hline CSF & Luminex assay & $\begin{array}{c}\text { There was no correlation between DJ-1 and striatal } \\
\text { dopaminergic function. }\end{array}$ & {$[23]$} \\
\hline Urine & WB & $\begin{array}{c}\text { DJ-1 levels in PD males were significantly higher than } \\
\text { those in controls. }\end{array}$ & {$[24]$} \\
\hline Saliva & WB & $\begin{array}{l}\text { DJ-1 was increased in PD and correlated with UPDRS } \\
\text { score. }\end{array}$ & {$[25]$} \\
\hline Urine & ELISA & OxiDJ-1 levels were higher in PD. & This study \\
\hline
\end{tabular}

${ }^{\mathrm{a}}$ Western blot analysis; ${ }^{\mathrm{b}}$ enzyme-linked immunosorbent assay.

TABLE 2: Summary of patient samples.

\begin{tabular}{lcccc}
\hline \multirow{2}{*}{ Gender } & \multicolumn{2}{c}{ Non-PD } & \multicolumn{2}{c}{$\mathrm{PD}^{\mathrm{a}}$} \\
& Male & Female & Male & Female \\
\hline Number & 6 & 16 & 15 & 18 \\
Age (years) & $71 \pm 11.3$ & $72 \pm 9.6$ & $76 \pm 4.2$ & $73 \pm 7.0$ \\
Onset duration (years) & $\mathrm{NA}^{\mathrm{b}}$ & $\mathrm{NA}^{\mathrm{b}}$ & $8.3 \pm 2.87$ & $7.3 \pm 1.87$ \\
\hline
\end{tabular}

${ }^{a} \mathrm{PD}$ patient information; PD diagnostic scores from the HY and UPDPS were not available; ${ }^{\mathrm{b}} \mathrm{NA}=$ not applicable.

in those of healthy subjects or medicated PD patients [13]. Intriguingly, MPTP, a drug that induces PD symptoms, increased OxiDJ-1 levels in mouse erythrocytes [14]. These evidences suggest that screening for oxidized DJ-1 levels in urine could be a convenient and efficient tool for diagnosing PD. As such, we established an enzyme-linked immunosorbent assay (ELISA) for examining levels of OxiDJ-1 in patient urine. Using this ELISA, we found significantly higher levels of OxiDJ-1 in $\mathrm{PD}$ patient urine compared to controls.

\section{Materials and Methods}

2.1. Cell Culture and Treatment. Human embryonic kidney 293T (HEK293T) cells were cultured in Dulbecco's medium (DMEM) high glucose (Cellgro, Herndon, VA, USA) supplemented with $10 \%$ fetal bovine serum (Cellgro) and $1 \%$ penicillin-streptomycin (GIBCO/BRL, Gaithersburg, MD, USA) for $24 \mathrm{~h}$ under $5 \% \mathrm{CO}_{2}$ conditions. We then treated the cells with $100 \mu \mathrm{M}$ of $\mathrm{H}_{2} \mathrm{O}_{2}$ (Sigma-Aldrich, St. Louis, $\mathrm{MO}$, USA) for 0,1 , and $3 \mathrm{~h}$. The culture media were collected, and cells were lysed directly in $1 \mathrm{X}$ sample buffer.

2.2. Patient Urine Sampling. Our study was approved by the Institutional Review Board of Sanbon Medical Center, Wonkwang University (IRB2013-24). Urine samples were collected from $33 \mathrm{PD}$ patients and 22 non-PD controls
(Table 2) at the Department of Neurology, Sanbon Medical Center. The PD assessments, the unified Parkinson's disease rating (UPDR), and Hoehn and Yahr (HY) scales, were not used in this study. Instead, all PD patients were diagnosed by a certified neurologist, based on the UK Brain Bank criteria. Before starting our analysis, we excluded urine samples with proteinuria, hematuria, or a $\mathrm{pH}$ lower than 5.5. Two milliliters of patient urine samples were thawed slowly overnight at $4^{\circ} \mathrm{C}$ and then vortexed. Samples were treated with $1 \%$ Triton X-100 and $1 \mathrm{X}$ Protease Inhibitor Cocktail Set III (Gendepot, Barker, TX, USA) and centrifuged at 18,000×g for $30 \mathrm{~min}$ at $4^{\circ} \mathrm{C}$. After centrifugation, the supernatant was concentrated by centrifugation of $10,000 \times \mathrm{g}$ for $30 \mathrm{~min}$ at $4^{\circ} \mathrm{C}$ using a NANOSEP $3 \mathrm{kDa}$ OMEGA filter (Pall Life sciences, Ann Arbor, MI, USA) prewashed with DBPS until the samples were concentrated 5 -fold.

2.3. Western Blot Assay. For our Western blot assay, $40 \mu \mathrm{l}$ of concentrated patient urine was mixed with $10 \mu \mathrm{l}$ of $5 \mathrm{X}$ protein sample buffer and boiled at $95^{\circ} \mathrm{C}$ for $5 \mathrm{~min}$. We followed the Western blot procedure as previously described [24]. All membranes were incubated with the following primary antibodies: anti-DJ-1 (1:1000, \#2134, Cell signaling technology, Danvers, MA, USA), anti-Oxidized DJ-1 (1:500, ab169520, MJF-R16(66-5), Abcam, Cambridge, MA, USA), anti-TSG 101 (1:1000, ab83, Abcam), and anti- $\beta$-actin $(1: 1000$, sc-47778, Santa Cruz, Dallas, TX, USA). The membranes were then incubated in goat anti-rabbit or -mouse IgG with horseradish peroxidase $(1: 5000$, Jackson Immunoresearch, West grove, PA, USA. 111-035-003 or 115-035-003, resp.). Intensity of the bands was measured by the Multi Gauge V 3.0 program (Fuji photo Film, Tokyo, Japan).

2.4. Sandwich Enzyme-Linked Immunosorbent Assay (ELISA). Recombinant His DJ-1 (His-DJ-1) was purchased 


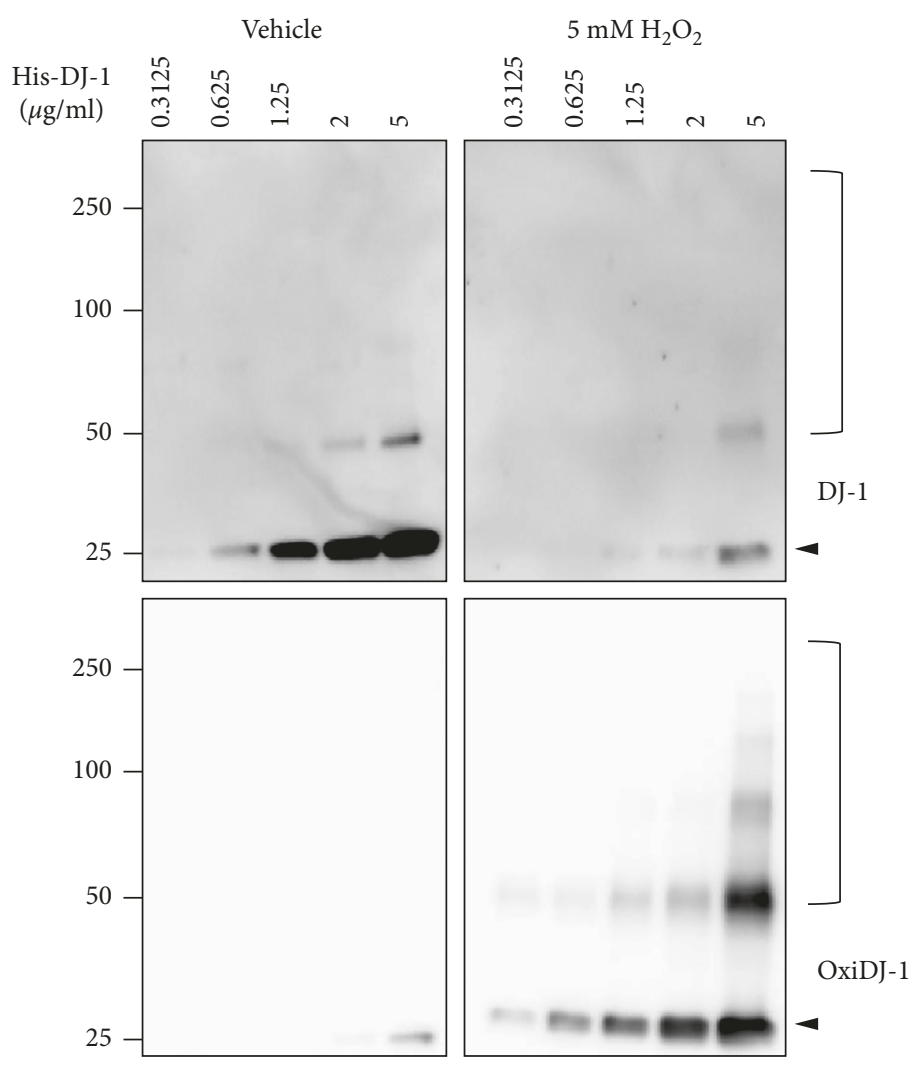

(a)
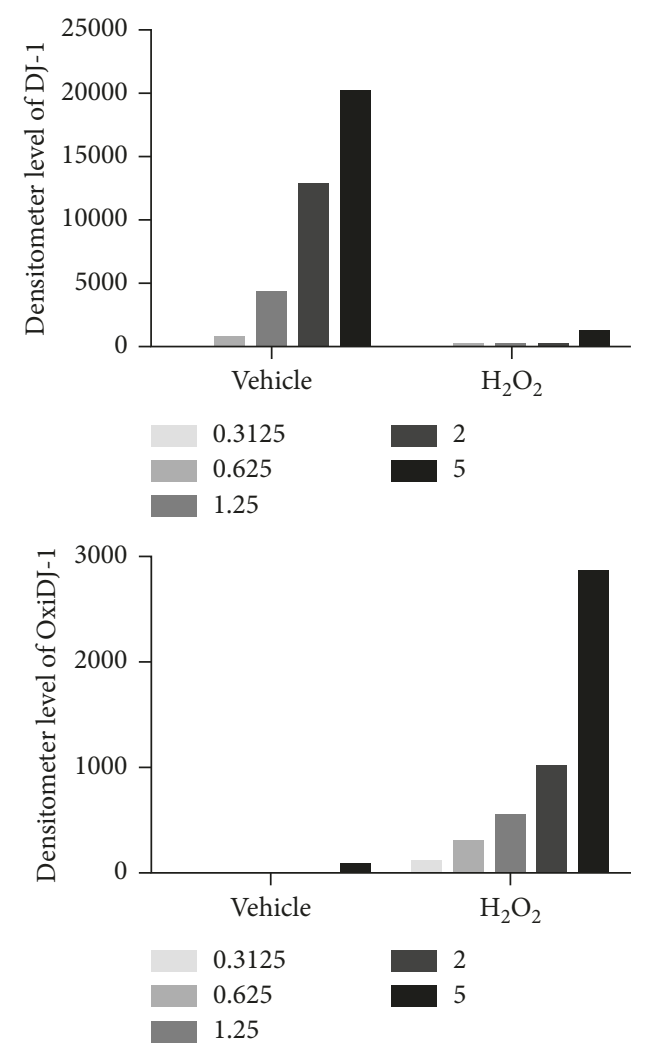

(b)

Figure 1: Continued. 

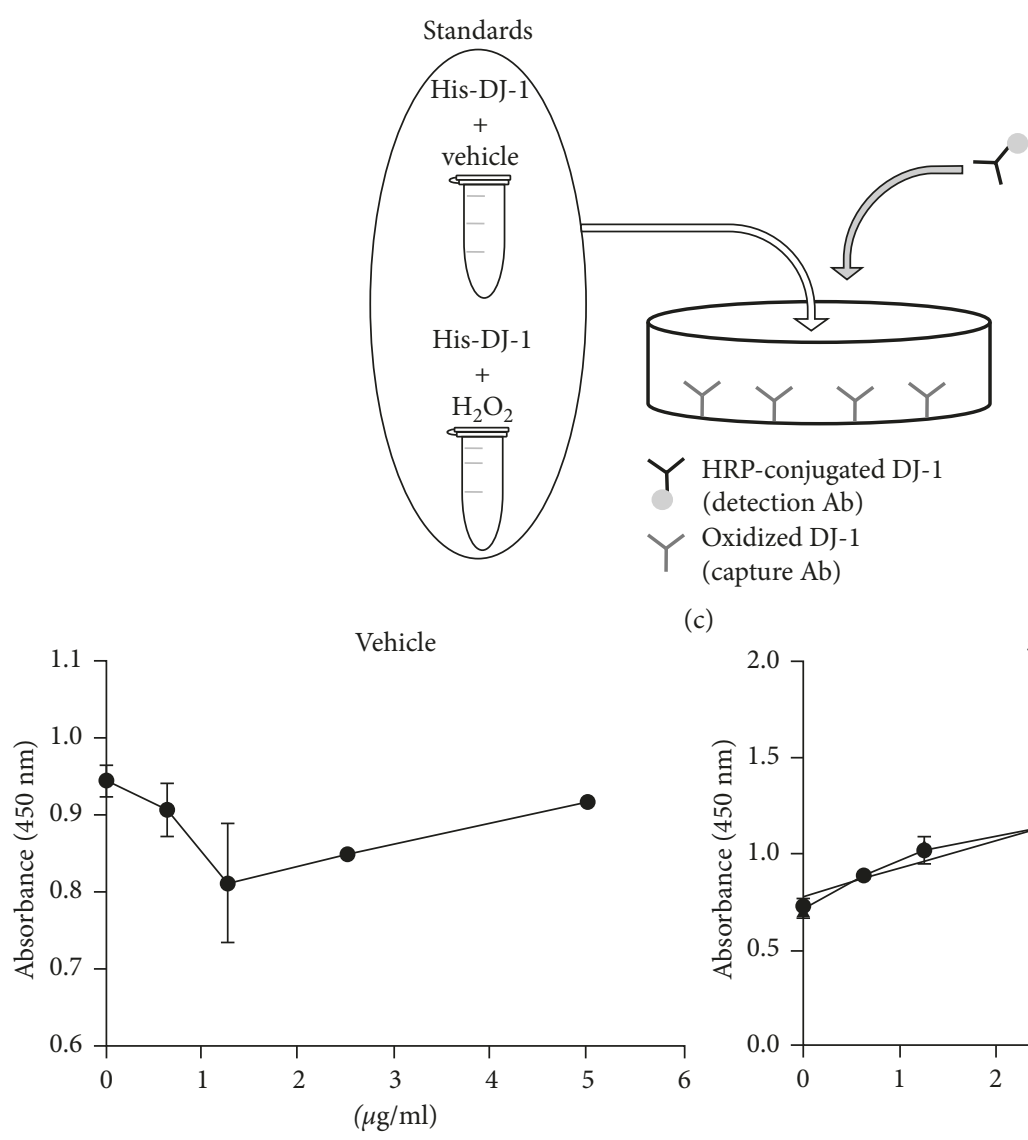

(c)

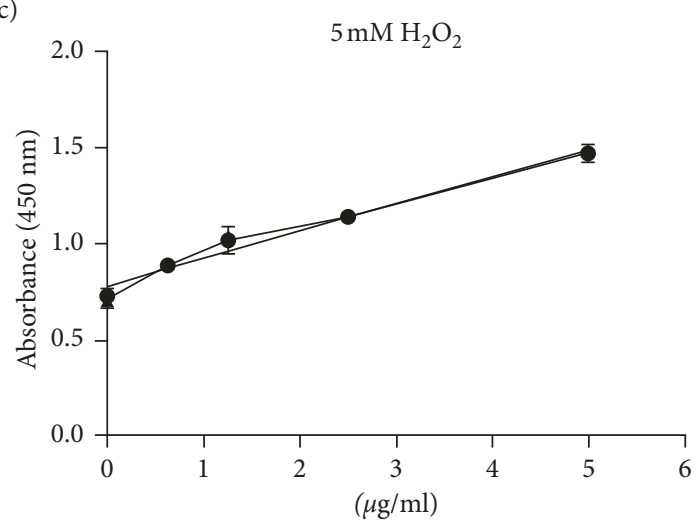

(d)

Figure 1: Establishment of the OxiDJ-1 ELISA. (a) Western blot analysis of OxiDJ-1. To generate OxiDJ-1, His-DJ-1 was incubated with $5 \mathrm{mM} \mathrm{H}_{2} \mathrm{O}_{2}$ or equivalent vehicle (sterile DW) for $1.5 \mathrm{~h}$ at $37^{\circ} \mathrm{C}$ and subjected to the Western blot analysis. (b) A quantitative analysis using a densitometer of the Western blot results in high molecular weight (HMW), or monomer was indicated by closed bracket or arrowhead, respectively. (c) A schematic diagram representing our OxiDJ-1 ELISA. (d) The standard curve obtained from the OxiDJ-1 ELISA. The ELISA was performed with an oxidized DJ-1 antibody as a capture antibody and HRP-conjugated DJ-1 antibody as a detection antibody using His-DJ-1 pretreated with $\mathrm{H}_{2} \mathrm{O}_{2}$ or DW as standard proteins.

from Sino biological (Beijing, China). To generate OxiDJ-1, His-DJ-1 was incubated with $5 \mathrm{mM} \mathrm{H}_{2} \mathrm{O}_{2}$ or equivalent vehicle (sterile distilled water (DW)) for $1.5 \mathrm{~h}$ at $37^{\circ} \mathrm{C}$. The generated OxiDJ-1 was confirmed by Western blot assay and standards of His-DJ-1 with $\mathrm{H}_{2} \mathrm{O}_{2}$ which were freshly made just before ELISA. For ELISA to detect OxiDJ-1, we coated each well of a MaxiSorp flat-bottom 96 well plate (44-240421, Nunc, Roskilde, Denmark) with OxiDJ-1 antibody $(0.5 \mu \mathrm{g} / \mathrm{ml})$ and $50 \mathrm{mM}$ carbonate buffer overnight at $4^{\circ} \mathrm{C}$. Each well was then washed 3 times with $200 \mu \mathrm{l}$ of 1 X PBST and blocked with $150 \mu \mathrm{l}$ of SuperBlock T20 (Thermo Fisher Scientific, Waltham, MA, USA) for $1 \mathrm{~h}$ at RT on a shaker. After blocking, each well was washed 4 times with $200 \mu$ l of PBST. Next, standard OxiDJ-1, patient and control urine samples were loaded into duplicate wells of the plate and incubated overnight at $4^{\circ} \mathrm{C}$ on a shaker. The wells were washed 4 times and then incubated in $100 \mu \mathrm{l}$ of DJ-1 antibody conjugated with HRP $(0.7 \mu \mathrm{g} / \mathrm{ml}, 60 \mathrm{R}-2218$, Fitzgerald Industries International, Concord, MA, USA) in SuperBlock T20 for $1.5 \mathrm{~h}$ at RT on a shaker. After that, the plate was washed 5 times with $200 \mu \mathrm{l}$ of PBST. We then added $100 \mu \mathrm{l}$ of $3,3^{\prime} 5,5^{\prime}$-tetramethylbenzidine (Sigma-Aldrich) into each well and incubated them for $10 \mathrm{~min}$ in a dark container at RT on a shaker. Lastly, $50 \mu \mathrm{l}$ of $2 \mathrm{~N} \mathrm{HCl}$ was added to each well, and the absorbance was measured by Synergy 2 (Biotek Instrument, Winooski, VT, USA) at a $450 \mathrm{~nm}$ wavelength.

2.5. Statistical Analysis. The graphs are presented as the mean \pm SEM. The data were analyzed using Prism6 (GraphPad software, La Jolla, CA, USA). Each statistical analysis is described in detail in the figure legends.

\section{Results}

3.1. Establishment of an OxiDJ-1 Sandwich ELISA Using Recombinant DJ-1 Protein Treated with $\mathrm{H}_{2} \mathrm{O}_{2}$. In order to establish an OxiDJ-1 sandwich ELISA, we generated $\mathrm{H}_{2} \mathrm{O}_{2}-$ mediated OxiDJ-1 by treatment of recombinant His-DJ-1 protein with $5 \mathrm{mM} \mathrm{H}_{2} \mathrm{O}_{2}$ for $1.5 \mathrm{~h}$ to use as a standard protein for ELISA. We confirmed that $\mathrm{H}_{2} \mathrm{O}_{2}$-treated His-DJ1 showed a distinct increase in OxiDJ-1 and a gradual 


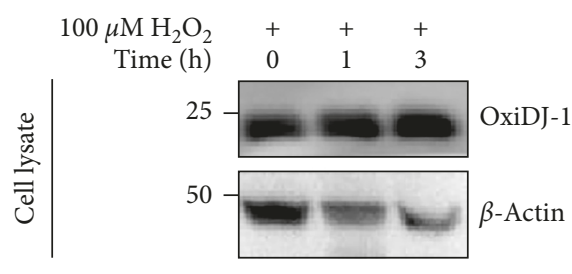

(a)

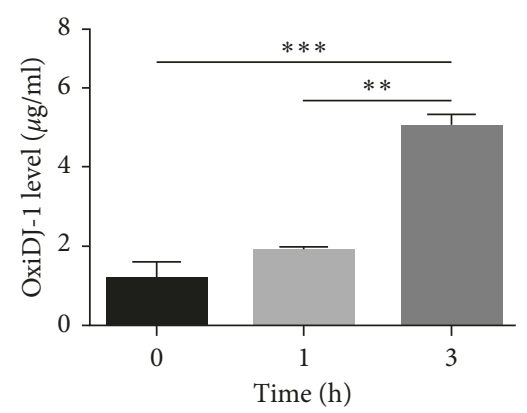

(c)

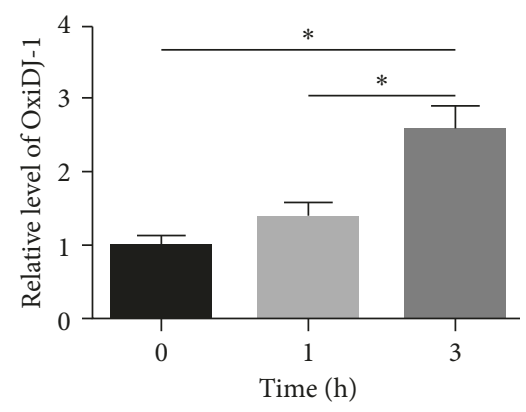

(e)

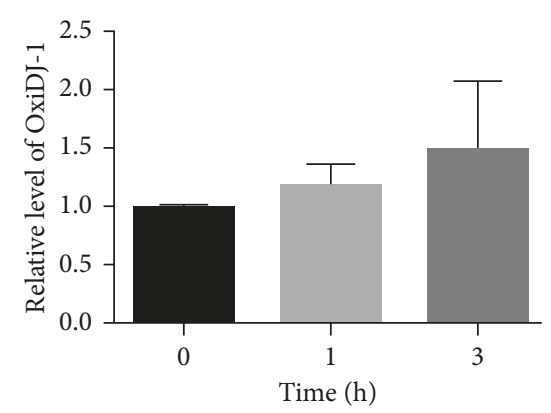

(b)

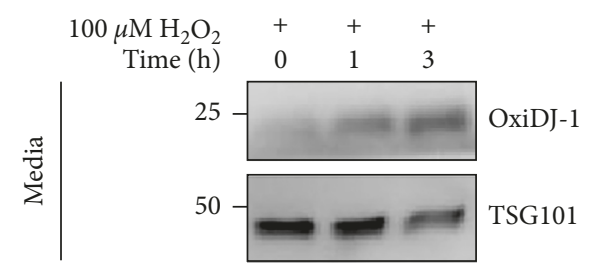

(d)

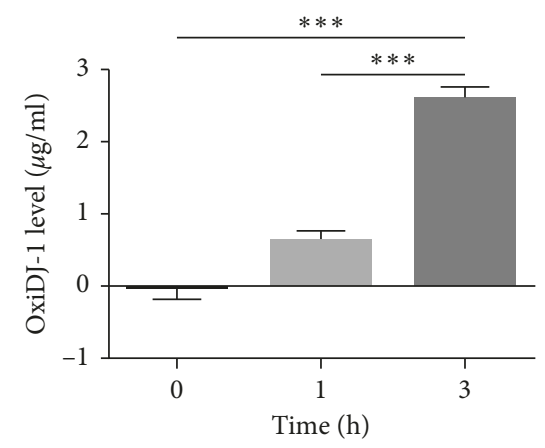

(f)

FIGUre 2: Detection of OxiDJ-1 treated with $\mathrm{H}_{2} \mathrm{O}_{2}$ in HEK293T cell lysates or cultured media. HEK293T cells were treated with $100 \mu \mathrm{M}$ $\mathrm{H}_{2} \mathrm{O}_{2}$ for 0,1 , and $3 \mathrm{~h}$, and their cell lysates (a) or culture media (d) were subjected to Western blot assay. A quantitative analysis of cellular (b) or media (e) OxiDJ-1 levels, which were normalized by their $\beta$-actin (b) or TSG101 (e) levels, was done. Increased OxiDJ-1 levels in cell lysates (c) or media (f) were detected by ELISA. $n=3$, duplication for ELISA, One-way ANOVA, ${ }^{*} p<0.05,{ }^{* *} p<0.01,{ }^{* * *} p<0.001$.

decrease in DJ-1 levels when DJ-1 concentration was gradually increased by Western blot analysis (Figures $1(\mathrm{a})$ and $1(\mathrm{~b})$ ). However, His-DJ-1 treated with DW, a vehicle control, had decreased or undetectable OxiDJ-1 levels (Figures 1(a) and 1(b)), while increased DJ-1 levels were detected. These data clearly confirm the activity of the OxiDJ-1 antibody. In addition, the formation of high-molecular weight DJ-1 aggregates seems to be facilitated by $\mathrm{H}_{2} \mathrm{O}_{2}$ treatment, as expected (Figure 1(a)).

We then designed a sandwich ELISA for OxiDJ-1, which was composed of oxidized DJ-1 and HRP-conjugated DJ-1 antibodies. Using the developed ELISA, we obtained an elaborate standard curve which had a gradual increase in $\mathrm{H}_{2} \mathrm{O}_{2}$-treated His-DJ-1 concentration, but not in DW-treated His-DJ-1 which had fluctuating absorbance (Figure 1(c)). These results suggest that our developed OxiDJ-1 sandwich ELISA could be a promising tool to measure OxiDJ-1 levels.
3.2. Validation of OxiDJ-1 Sandwich ELISA Using HEK293T Treated with $\mathrm{H}_{2} \mathrm{O}_{2}$. Previous studies have demonstrated that $\mathrm{H}_{2} \mathrm{O}_{2}$ treatment can induce OxiDJ-1 formation in the intracellular space and increase OxiDJ-1 levels in the extracellular space $[26,27]$. To induce the formation of OxiDJ-1 in cells, HEK293T cells were treated with $\mathrm{H}_{2} \mathrm{O}_{2}$ for the indicated times and OxiDJ-1 levels of both the cell lysates and culture media were measured by both Western blot and ELISA. In the Western blot assay, increased OxiDJ-1 levels were observed in cell-lysates in a time-dependent manner, though it was not statistically significant (Figures 2(a) and 2(b)). The OxiDJ-1 ELISA of the lysates showed significantly higher OxiDJ-1 levels after the $3 \mathrm{~h}$ treatment than after only the 0 or $1 \mathrm{~h}$ treatment (Figure 2(c)). Moreover, both Western blot and ELISA of the culture media exhibited significantly higher OxiDJ-1 levels in the $3 \mathrm{~h}$ treatment than the 0 or $1 \mathrm{~h}$ treatment 


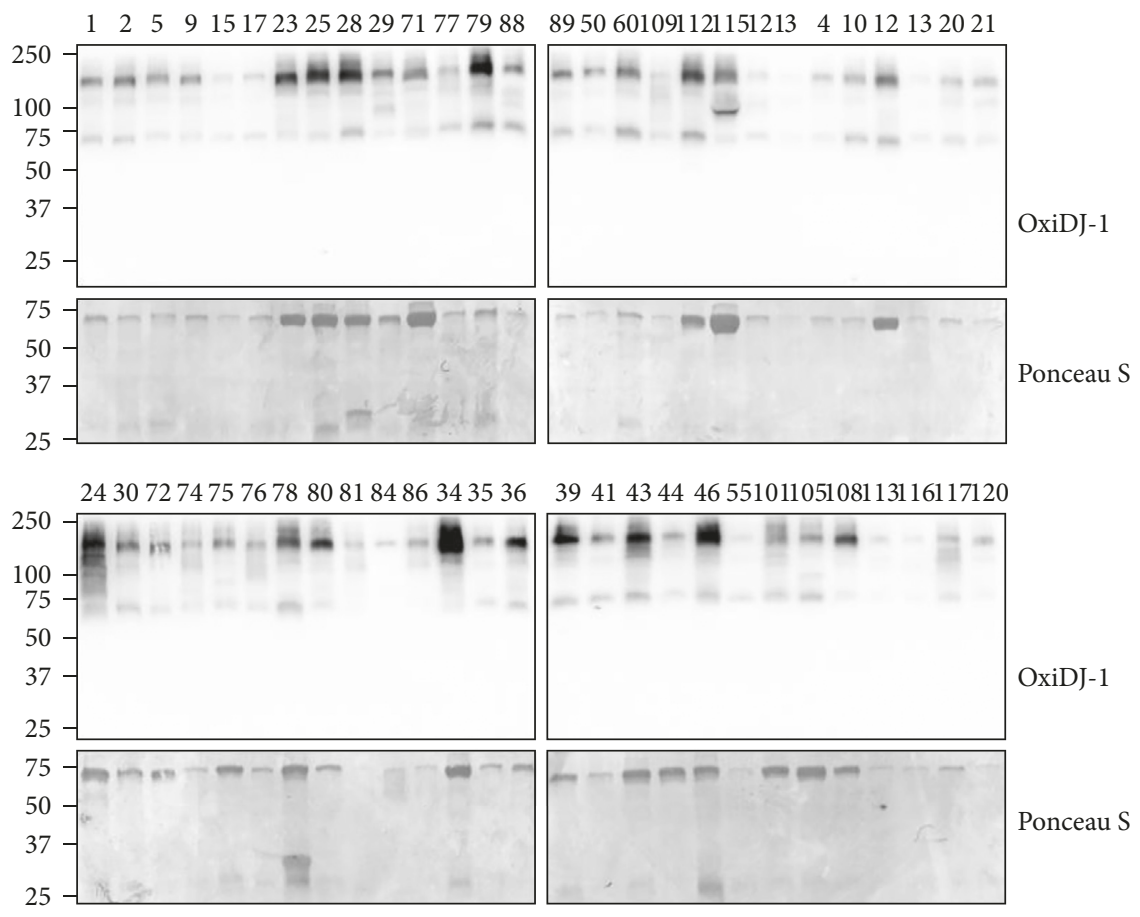

(a)

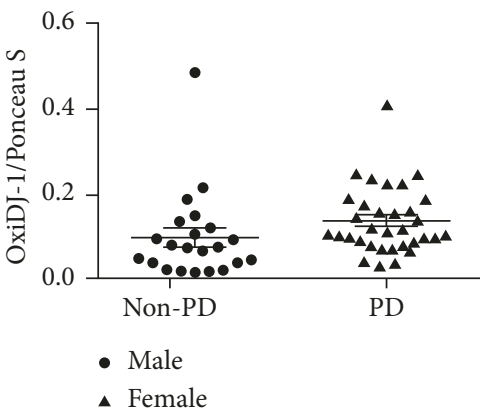

(b)

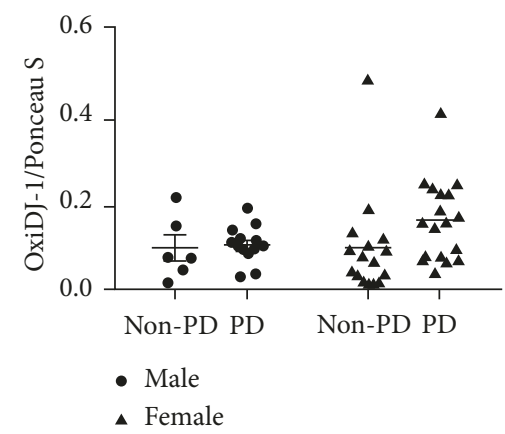

(c)

Figure 3: Comparison of OxiDJ-1 levels from patient urine samples using Western blot analysis. (a) Urine samples of non-PD controls (34, $35,36,39,41,43,44,46,50,55,60,101,105,108,109,112,113,115,116,117,120$, and 121) or PD patient $(1,2,3,4,5,9,10,12,13,15,17,20$, $21,23,24,25,28,29,30,71,72,74,75,76,77,78,79,80,81,84,86,88$, and 89) were subjected to Western blot. The numbers at the top of the images are the patient numbers. (b) OxiDJ-1 levels were normalized with total protein levels determined by Ponceau S staining, and their ratio was diagramed. Student's $t$-test was used for this analysis. (c) Gender-grouped OxiDJ-1 levels were analyzed. All comparisons with oneway ANOVA were not significant.

(Figures 2(d)-2(f)). These data support that our OxiDJ-1 ELISA is sensitive enough to detect live cell-derived OxiDJ-1. In addition, our results suggest that OxiDJ-1 is secreted in the culture medium.

\subsection{Investigations of OxiDJ-1 Levels in Urine Samples of} Korean PD Patients. Next, we tested the presence of OxiDJ-1 in human urine. A previous study used proteomic analysis to confirm that DJ-1 is secreted in human urine via exosomes [28]. Another study reported the presence of OxiDJ-1 in RBCs of human PD patients [13]. However, to date, there are no reports on the presence of OxiDJ-1 in human biofluids. In our study, Western blot assays of non-PD control or PD patient urine samples detected OxiDJ-1, but there was no significant difference in the amount of OxiDJ-1 between PD and non-PD participants (Figures 3(a) and 3(b)). Even after looking for gender-specific relevance within the two groups, we did not find any distinct differences (Figure 3(c)). Strikingly, when we used the established OxiDJ-1 ELISA, we found that PD patients had significantly 2-fold higher levels of OxiDJ-1 levels in their urine than non-PD controls (Figure 4(a)). Thus, our data confirms the presence of OxiDJ-1 in human urine.

There was also no significant gender-specific relevance within patient groups in OxiDJ-1 ELISA (Figure 4(b)). We also found no correlation between OxiDJ-1 level and PD onset age or duration (Supplementary Figure 1). We found some samples with negative quantities of OxiDJ-1 measuring at an absorbance lower than the interpolated standard of zero. This might be due to the formation of precipitates in their samples 


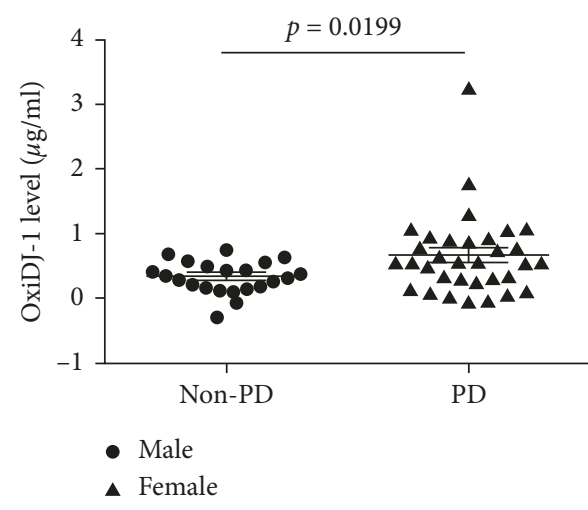

(a)

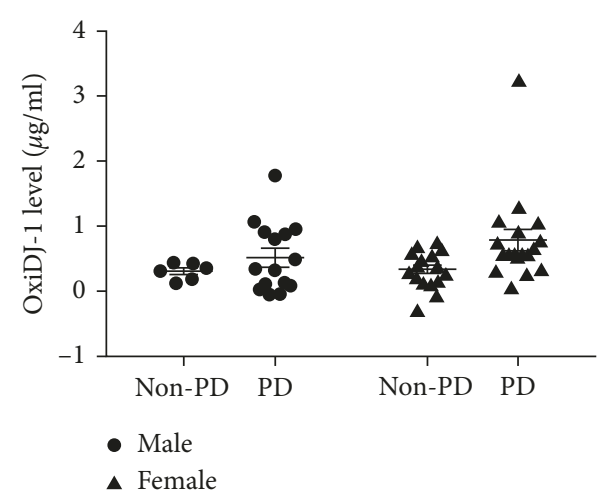

(b)

Figure 4: Detection of OxiDJ-1 from urine samples using ELISA. (a) OxiDJ-1 levels in urine were significantly increased in PD patients compared to non-PD controls. Student's $t$-test was used for this analysis, and $p$ value is described in the graph. (b) OxiDJ-1 levels in urine were divided by gender. One-way ANOVA was used for statistical analysis.

during ELISA, which might have reduced their antigenantibody reaction or interrupted the OxiDJ-1 binding to the capture antibody. Taken together, our results support that investigating OxiDJ-1 levels in human urine samples could serve as a putative PD diagnostic tool. To clarify our findings, further studies with a larger number of samples are necessary.

\section{Discussion}

Oxidative stress is a highly evaluated risk factor of PD progression [1]. Increased ROS in dopaminergic neurons can modify cytosolic proteins via chemical conjugation [4]. HNE is produced from oxidation of lipids with polyunsaturated omega- 6 acyl groups, such as arachidonic or linoleic acid, by lipid peroxidase $[2,29]$. It has been implicated that HNE is conjugated with $\alpha$-syn, thereby generating HNE-modified $\alpha$-syn oligomers [30, 31]. Furthermore, PD patients showed higher levels of HNE-modified $\alpha$-syn oligomers in the substantia nigra (SN) $[32,33]$. Previous studies have reported that extensive oxidation of lipofuscin could aggravate proteasome activity and cause cytosolic aggregates [34, 35]. Inhibited proteasome activity is associated with $\alpha$-synucleinopathy [36]. Other studies have demonstrated that DAQ can conjugate with $\alpha$-syn to form unstructured adducts, and further, increased DAQ can exacerbate the survival of PC12, a rat dopaminergic neuronal cell line, via mitochondrial dysfunction $[37,38]$. This evidence suggests that removal of oxidative stress via ROS-sensing molecules, such as chaperones, heat shock proteins, and DJ-1, is the most adequate therapeutic strategy for PD. In addition, cellular or extracellular OxiDJ-1 levels could be utilized as an indicator of oxidative stress levels.

As summarized in Table 1, numerous studies have investigated the use of DJ-1 as a PD biomarker. Several studies showed that DJ-1 levels in CSF $[15,18]$, plasma [16], and saliva [25] were increased in PD than the comparative groups. But other studies reported that DJ-1 levels were not different in serum samples from PD and control cases [17] and in CSF samples from parkinsonian syndrome and control groups [22]. On the other hand, contradictory levels of DJ-1 in CSF and saliva of PD were reported [20]. X. Lin et al. suggested that two types of HNE-modified DJ-1 isoform showed a distinguishable level in whole blood of PD [21]. Oxidative stressmediated HNE might be associated with oxidation of DJ-1. OxiDJ-1 increased in unmedicated PD than medicated PD or healthy group [13]. Considering the molecular function of DJ1 against oxidative stress, unmodified total DJ-1 might not be a suitable biomarker for PD, because the DJ-1 level showed inconsistent results depending on applications of biofluids or analytical methods. We assumed that oxidative stress-mediated modification of DJ-1 would be a hopeful approach for a diagnostic tool of PD.

Elimination of ROS by DJ-1 is accompanied by oxidation of DJ-1, itself [8]. A number of studies have shown that DJ-1 forms aggregates in the cytoplasm $[39,40]$. We also observed increased OxiDJ-1 aggregates after treatment of His-DJ-1 with $\mathrm{H}_{2} \mathrm{O}_{2}$ (Figure $1(\mathrm{a})$ ). Analyzing OxiDJ-1 levels in urine using Western blot assay also showed that most OxiDJ-1 in urine formed aggregates whereas monomeric OxiDJ-1 was barely detected after extreme long exposure (Figure 3(a)). We did not see any aggregated OxiDJ-1 or DJ-1 in cell lysates or media in the Western blot assay (data not shown). In HEK293T, OxiDJ-1 levels detected by the ELISA were consistent with those from the Western blot, but the differences were clearer in the ELISA (Figure 2). We only observed significant differences in OxiDJ-1 levels between $\mathrm{PD}$ and non-PD participants in the ELISA, not in the Western blot assay (Figures 3 and 4), suggesting that our ELISA is more sensitive than the Western blot assay.

Our previous study demonstrated that there were no significant differences in DJ-1 levels in urine exosomes during different times of day [24]. Further, only Korean male PD patients showed increased DJ-1 protein levels in their urine exosomes. However, DJ-1 proteins existed in both heated-soluble washing fractions, which are used for our analysis, and in retentate fractions, which were not analyzed in our previous study because of their low protein concentrations [24]. Therefore, in this study, we used the whole urine sample after incubating it with Triton X-100 detergent and concentrating it by filtration to acquire all proteins in the exosomes. This approach enabled us to detect 
the exact levels of total OxiDJ-1 in the urine sample of male and female patients. We were able to observe the presence of OxiDJ-1 in human urine and significant differences in OxiDJ-1 concentration between PD and non-PD participants. Although DJ-1 levels of Korean male PD patients were higher than those of female PD patients in our previous study, OxiDJ-1 levels exhibited no such difference (Supplementary Figure 2).

Consistent with previous reports, our study demonstrates that DJ-1 and OxiDJ-1 levels could be used as PD biomarkers in various materials from human. Using an OxiDJ-1 ELISA, we tried to observe differences in OxiDJ-1 levels in urine samples between PD patients and non-PD controls. However, it is important to note that in non-PD controls, OxiDJ-1 levels might be affected by other undiagnosed clinical conditions, including diabetes, hypertension, hyperlipidemia, and stroke. To verify the increase of OxiDJ-1 in PD patients, a future study with more PD patients and healthy, aged non-PD controls as well as healthy, young cohorts is necessary. Our finding might provide a key step towards finding an efficient diagnostic tool for PD.

\section{Conclusion}

We observed a 2-fold increase of OxiDJ-1 levels in the urine of Korean PD-patients compared to those in non-PD controls using our OxiDJ-1 ELISA. Thus, total OxiDJ-1 levels in human urine could be a feasible biomarker to diagnose PD.

\section{Conflicts of Interest}

The authors declare that they have no conflicts of interest.

\section{Acknowledgments}

The authors thank the staff in our neurology department and the donors and collaborators at the Inam Neuroscience Research Center. This paper was supported by Wonkwang University in 2016.

\section{Supplementary Materials}

Supplementary 1. Figure 1: correlation between onset duration of PD patients and OxiDJ-1 levels in patient urine. OxiDJ-1 levels were categorized by onset duration of PD. All onset duration groups were compared by one-way ANOVA and did not show any statistical differences.

Supplementary 2. Figure 2: comparison of OxiDJ-1 levels in urine by gender regardless of PD. OxiDJ-1 levels showed no statistical difference by Student's $t$-test between males and females.

\section{References}

[1] V. Dias, E. Junn, and M. M. Mouradian, "The role of oxidative stress in Parkinson's disease," Journal of Parkinson's disease, vol. 3, no. 4, pp. 461-491, 2013.

[2] H. Zhong and H. Yin, "Role of lipid peroxidation derived 4hydroxynonenal (4-HNE) in cancer: focusing on mitochondria," Redox Biology, vol. 4, pp. 193-199, 2015.
[3] B. S. Berlett and E. R. Stadtman, "Protein oxidation in aging, disease, and oxidative stress," Journal of Biological Chemistry, vol. 272, no. 33, pp. 20313-20316, 1997.

[4] E. R. Stadtman and B. S. Berlett, "Reactive oxygen-mediated protein oxidation in aging and disease," Chemical Research in Toxicology, vol. 10, no. 5, pp. 485-494, 1997.

[5] S. B. Berman and T. G. Hastings, "Dopamine oxidation alters mitochondrial respiration and induces permeability transition in brain mitochondria: implications for Parkinson's disease," Journal of Neurochemistry, vol. 73, no. 3, pp. 1127-1137, 1999.

[6] C. L. Pham, S. L. Leong, F. E. Ali et al., "Dopamine and the dopamine oxidation product 5,6-dihydroxylindole promote distinct on-pathway and off-pathway aggregation of alphasynuclein in a pH-dependent manner," Journal of Molecular Biology, vol. 387, no. 3, pp. 771-785, 2009.

[7] N. Lev, D. Roncevic, D. Ickowicz, E. Melamed, and D. Offen, "Role of DJ-1 in Parkinson's disease," Journal of Molecular Neuroscience, vol. 29, no. 3, pp. 215-225, 2006.

[8] M. A. Wilson, "The role of cysteine oxidation in DJ-1 function and dysfunction," Antioxidants and Redox Signaling, vol. 15, no. 1, pp. 111-122, 2011.

[9] Y. Saito, "Oxidized DJ-1 as a possible biomarker of Parkinson's disease," Journal of Clinical Biochemistry and Nutrition, vol. 54, no. 3, pp. 138-144, 2014.

[10] S. Batelli, D. Albani, R. Rametta et al., "DJ-1 modulates $\alpha$-synuclein aggregation state in a cellular model of oxidative stress: relevance for Parkinson's disease and involvement of HSP70," PLoS One, vol. 3, no. 4, article e1884, 2008.

[11] S. Shendelman, A. Jonason, C. Martinat, T. Leete, and A. Abeliovich, "DJ-1 is a redox-dependent molecular chaperone that inhibits a-synuclein aggregate formation," PLoS Biology, vol. 2, no. 11, p. e362, 2004.

[12] W. Zhou, M. Zhu, M. A. Wilson, G. A. Petsko, and A. L. Fink, "The oxidation state of $\mathrm{dj}-1$ regulates its chaperone activity toward a-synuclein," Journal of Molecular Biology, vol. 356, no. 4, pp. 1036-1048, 2006.

[13] Y. Saito, T. Hamakubo, Y. Yoshida et al., "Preparation and application of monoclonal antibodies against oxidized DJ-1. Significant elevation of oxidized DJ-1 in erythrocytes of earlystage Parkinson disease patients," Neuroscience Letters, vol. 465, no. 1, pp. 1-5, 2009.

[14] Y. O. Akazawa, Y. Saito, T. Hamakubo et al., "Elevation of oxidized DJ-1 in the brain and erythrocytes of Parkinson disease model animals," Neuroscience Letters, vol. 483, no. 3, pp. 201-205, 2010.

[15] M. Waragai, J. Wei, M. Fujita et al., "Increased level of DJ-1 in the cerebrospinal fluids of sporadic Parkinson's disease," Biochemical and Biophysical Research Communications, vol. 345, no. 3, pp. 967-972, 2006.

[16] M. Waragai, M. Nakai, J. Wei et al., "Plasma levels of DJ-1 as a possible marker for progression of sporadic Parkinson's disease," Neuroscience Letters, vol. 425, no. 1, pp. 18-22, 2007.

[17] C. Maita, S. Tsuji, I. Yabe et al., "Secretion of DJ-1 into the serum of patients with Parkinson's disease," Neuroscience Letters, vol. 431, no. 1, pp. 86-89, 2008.

[18] Z. Hong, M. Shi, K. A. Chung et al., "DJ-1 and alpha-synuclein in human cerebrospinal fluid as biomarkers of Parkinson's disease," Brain, vol. 133, no. 3, pp. 713-726, 2010.

[19] M. Shi, C. P. Zabetian, A. M. Hancock et al., "Significance and confounders of peripheral DJ-1 and alpha-synuclein in Parkinson's disease," Neuroscience Letters, vol. 480, no. 1, pp. 78-82, 2010.

[20] I. Devic, H. Hwang, J. S. Edgar et al., "Salivary alpha-synuclein and DJ-1: potential biomarkers for Parkinson's disease," Brain, vol. 134, no. 7, p. e178, 2011. 
[21] X. Lin, T. J. Cook, C. P. Zabetian et al., "DJ-1 isoforms in whole blood as potential biomarkers of Parkinson disease," Scientific Reports, vol. 2, no. 1, p. 954, 2012.

[22] L. Salvesen, S. Bech, A. Lokkegaard et al., "The DJ-1 concentration in cerebrospinal fluid does not differentiate among Parkinsonian syndromes," Parkinsonism and Related Disorders, vol. 18, no. 7, pp. 899-901, 2012.

[23] M. Shi, A. Furay, V. Sossi et al., "DJ-1 and aSYN in LRRK2 CSF do not correlate with striatal dopaminergic function," Neurobiology of Aging, vol. 33, no. 4, pp. 836.e835-836.e837, 2012.

[24] D. H. Ho, S. Yi, H. Seo, I. Son, and W. Seol, "Increased DJ-1 in urine exosome of Korean males with Parkinson's disease," BioMed Research International, vol. 2014, Article ID 704678, 8 pages, 2014

[25] J. M. Masters, A. J. Noyce, T. T. Warner et al., "Elevated salivary protein in Parkinson's disease and salivary DJ-1 as a potential marker of disease severity," Parkinsonism and Related Disorders, vol. 21, no. 10, pp. 1251-1255, 2015.

[26] S. Koide-Yoshida, T. Niki, M. Ueda et al., "DJ-1 degrades transthyretin and an inactive form of DJ-1 is secreted in familial amyloidotic polyneuropathy," International Journal of Molecular Medicine, vol. 19, no. 6, pp. 885-893, 2007.

[27] T. Kinumi, J. Kimata, T. Taira, H. Ariga, and E. Niki, "Cysteine-106 of DJ-1 is the most sensitive cysteine residue to hydrogen peroxide-mediated oxidation in vivo in human umbilical vein endothelial cells," Biochemical and Biophysical Research Communications, vol. 317, no. 3, pp. 722-728, 2004.

[28] E. J. Hoorn, T. Pisitkun, R. Zietse et al., "Prospects for urinary proteomics: exosomes as a source of urinary biomarkers," Nephrology, vol. 10, no. 3, pp. 283-290, 2005.

[29] K. Uchida, "4-Hydroxy-2-nonenal: a product and mediator of oxidative stress," Progress in Lipid Research, vol. 42, no. 4, pp. 318-343, 2003.

[30] Z. Qin, D. Hu, S. Han, S. H. Reaney, D. A. Di Monte, and A. L. Fink, "Effect of 4-hydroxy-2-nonenal modification on alpha-synuclein aggregation," Journal of Biological Chemistry, vol. 282, no. 8, pp. 5862-5870, 2007.

[31] E.-J. Bae, D.-H. Ho, E. Park et al., "Lipid peroxidation product 4-hydroxy-2-nonenal promotes seeding-capable oligomer formation and cell-to-cell transfer of a-synuclein," Antioxidants and Redox Signaling, vol. 18, no. 7, pp. 770-783, 2013.

[32] R. J. Castellani, G. Perry, S. L. Siedlak et al., "Hydroxynonenal adducts indicate a role for lipid peroxidation in neocortical and brainstem Lewy bodies in humans," Neuroscience Letters, vol. 319, no. 1, pp. 25-28, 2002.

[33] A. Yoritaka, N. Hattori, K. Uchida, M. Tanaka, E. R. Stadtman, and Y. Mizuno, "Immunohistochemical detection of 4hydroxynonenal protein adducts in Parkinson disease," Proceedings of the National Academy of Sciences of the United States of America, vol. 93, no. 7, pp. 2696-2701, 1996.

[34] A. Hohn, T. Jung, S. Grimm, B. Catalgol, D. Weber, and T. Grune, "Lipofuscin inhibits the proteasome by binding to surface motifs," Free Radical Biolology and Medicine, vol. 50, no. 5, pp. 585-591, 2011.

[35] A. Höhn and T. Grune, "Lipofuscin: formation, effects and role of macroautophagy()," Redox Biology, vol. 1, no. 1, pp. 140-144, 2013.

[36] H. J. Rideout, K. E. Larsen, D. Sulzer, and L. Stefanis, "Proteasomal inhibition leads to formation of ubiquitin/ alpha-synuclein-immunoreactive inclusions in PC12 cells," Journal of Neurochemistry, vol. 78, no. 4, pp. 899-908, 2001.

[37] M. Bisaglia, L. Tosatto, F. Munari et al., "Dopamine quinones interact with alpha-synuclein to form unstructured adducts,"
Biochemical and Biophysical Research Communications, vol. 394, no. 2, pp. 424-428, 2010.

[38] H. Q. Liu, X. Z. Zhu, and E. Q. Weng, "Intracellular dopamine oxidation mediates rotenone-induced apoptosis in PC12 cells," Acta pharmacologica Sinica, vol. 26, no. 1, pp. 17-26, 2005.

[39] S. Baulac, M. J. LaVoie, J. Strahle et al., "Dimerization of Parkinson's disease-causing DJ-1 and formation of high molecular weight complexes in human brain," Molecular and Cellular Neurosciences, vol. 27, no. 3, pp. 236-246, 2004.

[40] K. G. Shadrach, M. E. Rayborn, J. G. Hollyfield et al., "DJ-1dependent regulation of oxidative stress in the retinal pigment epithelium (RPE)," PLoS one, vol. 8, no. 7, article e67983, 2013. 


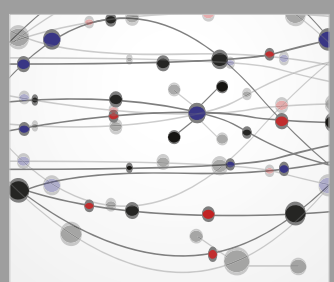

The Scientific World Journal
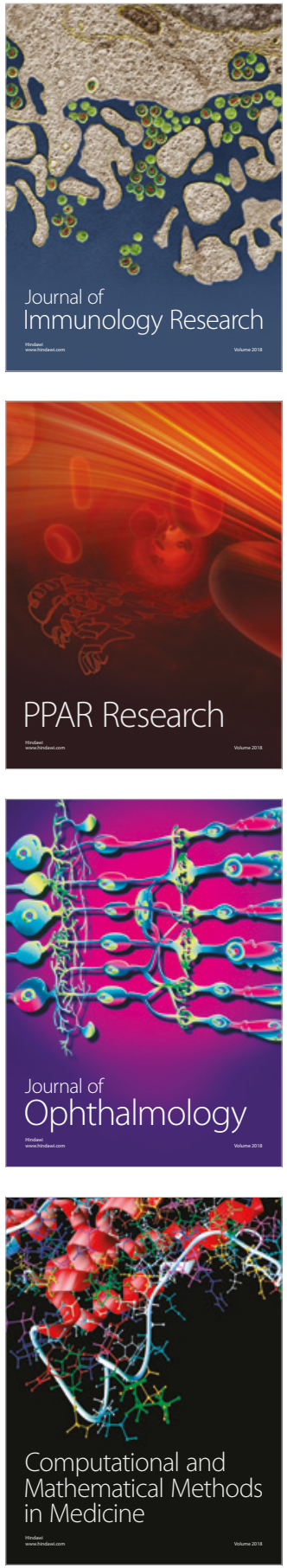

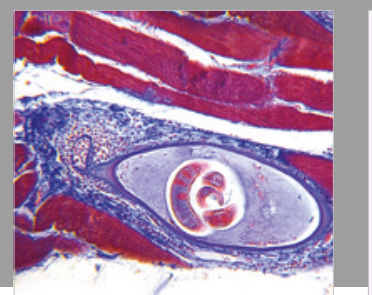

Gastroenterology Research and Practice

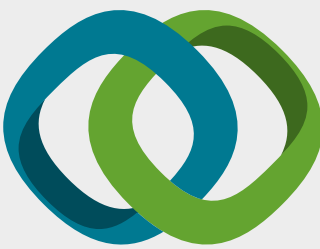

\section{Hindawi}

Submit your manuscripts at

www.hindawi.com
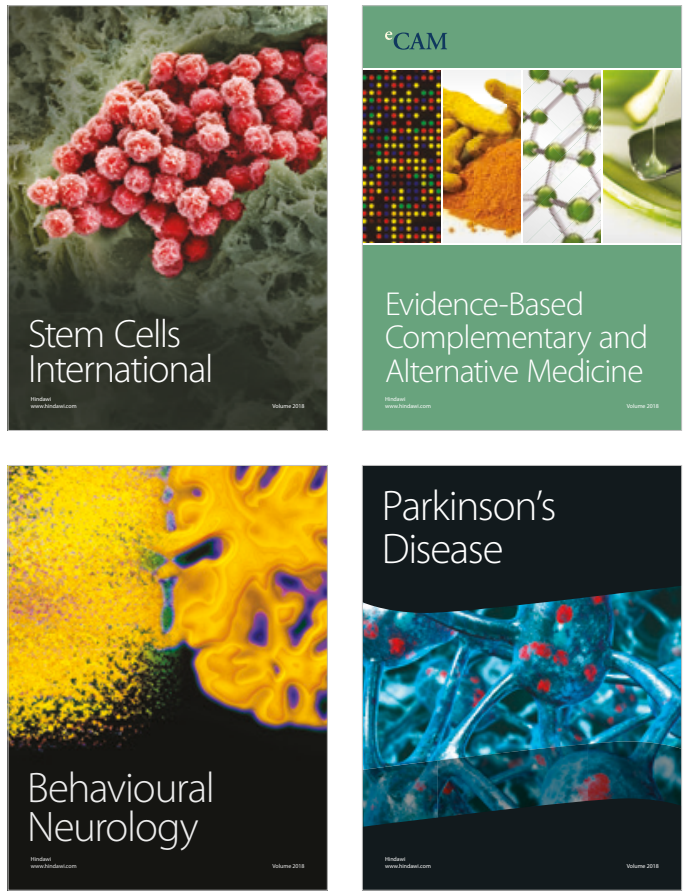

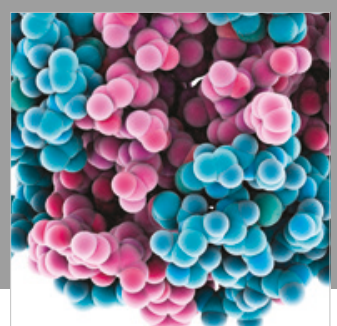

ournal of

Diabetes Research

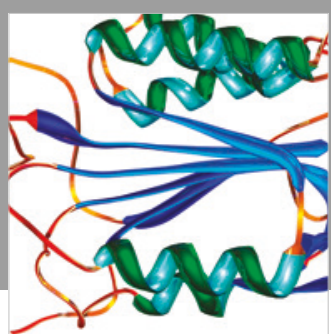

Disease Markers
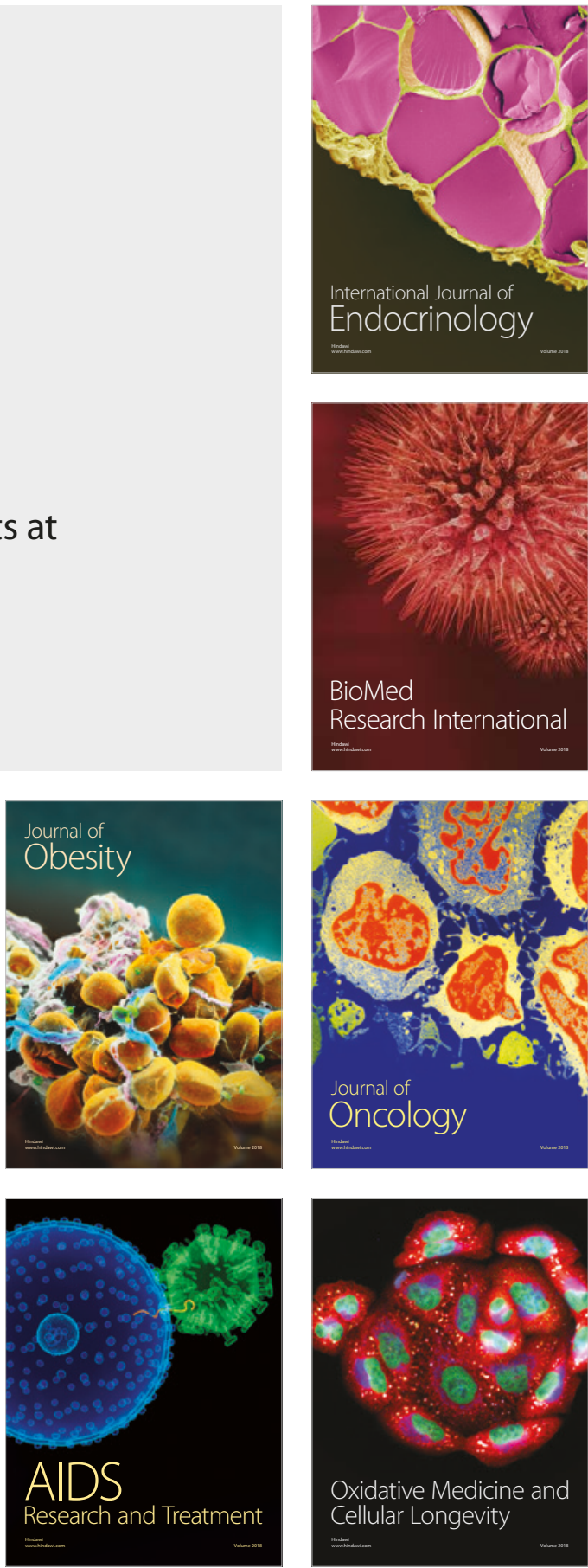